\title{
Fate of the Tetraquark Candidate Zc(3900) from Lattice QCD
}

\section{AUTHOR(S):}

Ikeda, Yoichi; Aoki, Sinya; Doi, Takumi; Gongyo, Shinya; Hatsuda, Tetsuo; Inoue, Takashi; Iritani, Takumi; ... Murano, Keiko; Sasaki, Kenji; HAL QCD Collaboration

\section{CITATION:}

Ikeda, Yoichi ... [et al]. Fate of the Tetraquark Candidate Zc(3900) from Lattice QCD. Physical Review Letters 2016, 117(24): 242001.

\section{ISSUE DATE:}

2016-12-09

URL:

http://hdl.handle.net/2433/250356

RIGHT:

C 2016 American Physical Society 


\title{
Fate of the Tetraquark Candidate $Z_{c}(3900)$ from Lattice QCD
}

\author{
Yoichi Ikeda, ${ }^{1,2}$ Sinya Aoki, ${ }^{3,4}$ Takumi Doi, ${ }^{2}$ Shinya Gongyo, ${ }^{3}$ Tetsuo Hatsuda, ${ }^{2,5}$ Takashi Inoue, \\ Takumi Iritani, ${ }^{7}$ Noriyoshi Ishii, ${ }^{1}$ Keiko Murano, ${ }^{1}$ and Kenji Sasaki ${ }^{3,4}$ \\ (HAL QCD Collaboration) \\ ${ }^{1}$ Research Center for Nuclear Physics (RCNP), Osaka University, Osaka 567-0047, Japan \\ ${ }^{2}$ Theoretical Research Division, Nishina Center, RIKEN, Saitama 351-0198, Japan \\ ${ }^{3}$ Yukawa Institute for Theoretical Physics, Kyoto University, Kyoto 606-8502, Japan \\ ${ }^{4}$ Center for Computational Sciences, University of Tsukuba, Ibaraki 305-8571, Japan \\ ${ }^{5}$ iTHES Research Group, RIKEN, Saitama 351-0198, Japan \\ ${ }^{6}$ Nihon University, College of Bioresource Sciences, Kanagawa 252-0880, Japan \\ ${ }^{7}$ Department of Physics and Astronomy, Stony Brook University, New York 11794-3800, USA
}

(Received 25 February 2016; revised manuscript received 15 June 2016; published 9 December 2016)

The possible exotic meson $Z_{c}(3900)$, found in $e^{+} e^{-}$reactions, is studied by the method of coupledchannel scattering in lattice QCD. The interactions among $\pi J / \psi, \rho \eta_{c}$, and $\bar{D} D^{*}$ channels are derived from (2+1)-flavor QCD simulations at $m_{\pi}=410-700 \mathrm{MeV}$. The interactions are dominated by the offdiagonal $\pi J / \psi-\bar{D} D^{*}$ and $\rho \eta_{c}-\bar{D} D^{*}$ couplings, which indicates that the $Z_{c}(3900)$ is not a usual resonance but a threshold cusp. Semiphenomenological analyses with the coupled-channel interaction are also presented to confirm this conclusion.

DOI: 10.1103/PhysRevLett.117.242001

One of the long-standing problems in hadron physics is to identify the existence of exotic hadrons different from the quark-antiquark states (mesons) and three-quark states (baryons). Candidates of such exotic hadrons include the pentaquark states $P_{c}^{+}(4380)$ and $P_{c}^{+}(4450)$ observed by the LHCb Collaboration [1] and the tetraquark states $Z_{c}(3900)$ reported by the BESIII [2], Belle [3], and CLEO-c [4] Collaborations. In particular, $Z_{c}(3900)$ appears as a peak in both the $\pi^{ \pm} J / \psi$ and $\bar{D} D^{*}$ invariant mass spectra in the reaction, $e^{+} e^{-} \rightarrow Y(4260) \rightarrow \pi^{ \pm} \pi^{\mp} J / \psi, \pi \bar{D} D^{*}$ : Its quantum numbers are then identified as $I^{G}\left(J^{P C}\right)=1^{+}\left(1^{+-}\right)$, so that at least four quarks, $c \bar{c} u \bar{d}$ (or its isospin partners), are involved. (See the level structure and the decay scheme in Fig. 1).

So far, there have been various phenomenological attempts to characterize the $Z_{c}(3900)$ as a hadrocharmonium, a compact tetraquark, a hadronic molecule (e.g., Refs. [5,6]) as well as a kinematical threshold effect (e.g., Refs. [7,8]). However, due to the lack of information of the diagonal and off-diagonal interactions among different channels (such as $\pi J / \psi, \rho \eta_{c}$, and $\bar{D} D^{*}$ ), the predictions of those models are not well under theoretical control. On the other hand, the direct lattice QCD studies with the standard method of temporal correlations show no candidate for the $Z_{c}(3900)$ eigenstate $[9,10]$, which indicates that the $Z_{c}(3900)$ may not be an ordinary resonance state. Under these circumstances, it is most desirable to carry out manifestly coupled-channel analyses with the firstprinciples QCD inputs.
The purpose of this Letter is to report a first attempt to determine the nature of the $Z_{c}(3900)$ on the basis of the HAL QCD method [11-14]. We consider three two-body channels below $Z_{c}(3900)\left(\pi J / \psi, \rho \eta_{c}\right.$, and $\left.\bar{D} D^{*}\right)$ which couple with each other. The interactions among these channels faithful to the QCD $S$ matrix are derived from the equal-time Nambu-Bethe-Salpeter (NBS) wave functions on the lattice according to the coupled-channel formulation of the HAL QCD method [15-17]. The $s$-wave interactions and the $S$ matrix thus obtained are used to search for the complex poles in the $\pi J / \psi$

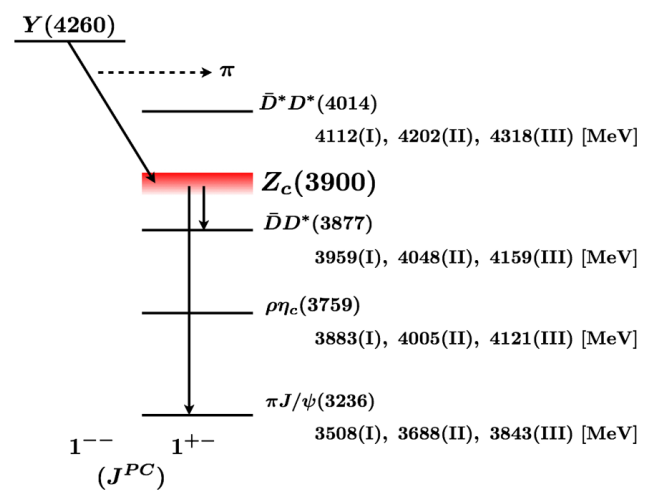

FIG. 1. A possible decay scheme of the $Y(4260)$ through $Z c$ (3900), together with the relevant two-meson thresholds of the $Z_{c}(3900)$ decay at $m_{\pi} \simeq 140$ (Expt.), 410 (case I), 570 (case II), and 700 (case III) MeV. The arrows represent the observed decay modes in the experiments [2-4]. 
and $\bar{D} D^{*}$ scattering amplitudes to unravel the nature of the $Z_{c}(3900)$. We note here that the conventional resonances such as the $\rho$ meson and the $\Delta$ baryon have not yet been analyzed in the HAL QCD method, and the comparison with the Lüscher's method [18] in these channels is one of the important future subjects. (Such comparison in the nonresonant $\pi \pi$ channel has been done in Refs. $[19,20]$; see also Ref. [21]). Note also that the coupled-channel HAL QCD method has not been experimentally tested in other systems yet: Baryon-baryon interactions with hyperons are currently underway [22] for comparison with future experimental data. With these reservations in mind, we extract invariant mass spectra of the three-body decays $Y(4260) \rightarrow$ $\pi \pi J / \psi$ and $\pi \bar{D} D^{*}$ using the scattering amplitudes obtained in lattice QCD, and the results are then compared with experimental data.

The starting point of the coupled-channel HAL QCD method [15-17] is a normalized correlation function

$$
C^{\alpha \beta}(\vec{r}, t) \equiv \sum_{\vec{x}}\left\langle 0\left|\phi_{1}^{\alpha}(\vec{x}+\vec{r}, t) \phi_{2}^{\alpha}(\vec{x}, t) \overline{\mathcal{J}}^{\beta}\right| 0\right\rangle / \sqrt{Z_{1}^{\alpha} Z_{2}^{\alpha}},
$$

where each channel is specified by $\alpha=\left(\pi J / \psi, \rho \eta_{c}, \bar{D} D^{*}\right)$, and $\phi_{i}^{\alpha}(\vec{y}, t)$ is a local Heisenberg operator at Euclidian time $t>0$ and the spatial point $\vec{y}$ for the meson $i(=1,2)$ with mass $m_{i}^{\alpha}$ in channel $\alpha$. The corresponding wave function renormalization factor is given by $Z_{i}^{\alpha}$. $\overline{\mathcal{J}}^{\beta}$ denotes a twomeson operator in channel $\beta$ with a zero-momentum wall quark source located at $t=0$. The NBS wave function $\psi_{n}^{\alpha}(\vec{r})$ for each scattering state specified by $n$ on the lattice is related to Eq. (1) as $C^{\alpha \beta}(\vec{r}, t)=\sum_{n} \psi_{n}^{\alpha}(\vec{r}) A_{n}^{\beta} e^{-W_{n} t}$ with $W_{n}$ being the eigenvalue of the $n$th QCD eigenstate. $A_{n}^{\beta} \equiv$ $\left\langle W_{n}\left|\overline{\mathcal{J}}^{\beta}\right| 0\right\rangle$ is an overlap between the eigenstate and QCD vacuum by the insertion of $\bar{J}^{\beta}$. It can be shown that $R^{\alpha \beta}(\vec{r}, t) \equiv C^{\alpha \beta}(\vec{r}, t) e^{\left(m_{1}^{\alpha}+m_{2}^{\alpha}\right) t}$ satisfies the Schrödingertype equation $[13,16]$,

$$
\begin{aligned}
& \left(-\frac{\partial}{\partial t}-H_{0}^{\alpha}\right) R^{\alpha \beta}(\vec{r}, t) \\
& =\sum_{\gamma} \Delta^{\alpha \gamma} \int d \overrightarrow{r^{\prime}} U^{\alpha \gamma}\left(\vec{r}, \vec{r}^{\prime}\right) R^{\gamma \beta}\left(\vec{r}^{\prime}, t\right),
\end{aligned}
$$

where $H_{0}^{\alpha}=-\nabla^{2} / 2 \mu^{\alpha}$ with the reduced mass $\mu^{\alpha}=$ $m_{1}^{\alpha} m_{2}^{\alpha} /\left(m_{1}^{\alpha}+m_{2}^{\alpha}\right)$ and $\Delta^{\alpha \gamma}=e^{\left(m_{1}^{\alpha}+m_{2}^{\alpha}\right) t} / e^{\left(m_{1}^{\gamma}+m_{2}^{\gamma}\right) t}$. In the above equation, we neglect terms associated with relativistic corrections, $\mathcal{O}\left(\left(\partial_{t}^{2} / m_{1,2}^{\alpha}\right)\left(\partial_{t} / m_{1,2}^{\alpha}\right)^{n}\right)$ with $n \geq 0$. We have checked that the relativistic corrections are negligible in the present lattice setup with relatively large pion masses. Here we consider $t$ sufficiently large so that the inelastic states (The lowest one is $\bar{D}^{*} D^{*}$ in the present lattice QCD setup) becomes negligible in $U^{\alpha \beta}$, otherwise these channels should be taken into account explicitly. The energy-independent coupled-channel potential $U^{\alpha \beta}\left(\vec{r}, \overrightarrow{r^{\prime}}\right)$ guarantees that the $S$ matrix is unitary below the $\bar{D}^{*} D^{*}$ threshold $[15,16]$ and gives the correct scattering amplitude. In the following, we take the $s$-wave projection ( $A_{1}^{+}$projection of the cubic group on the lattice) and also employ the lowest order of the velocity expansion, $U^{\alpha \beta}\left(\vec{r}, \overrightarrow{r^{\prime}}\right)=V^{\alpha \beta}(\vec{r}) \delta\left(\vec{r}-\overrightarrow{r^{\prime}}\right)+O\left(\nabla^{2}\right)$ to extract the spherical and local potential $V^{\alpha \beta}(r)$. The systematic errors originating from higher derivative terms are estimated by the $t$ dependence of the observables [13].

We note here that the HAL QCD method and the conventional Lüscher's method are both based on Eq. (1). In the coupled-channel Lüscher's method proposed in Refs. [23-25], some phenomenological parametrization of the $K$ matrix is employed that approximates the energy dependence of the coupled-channel $S$ matrix, while in the present method, the velocity expansion is employed that approximates the nonlocality of the coupled-channel potentials.

In order to extract $V^{\alpha \beta}(r)$ from lattice QCD simulation, we employ $(2+1)$-flavor QCD gauge configurations generated by the PACS-CS Collaboration [26,27] on a $32^{3} \times 64$ lattice with the renormalization group improved gauge action at $\beta_{\text {lat }}=1.90$ and the nonperturbatively $O(a)$-improved Wilson quark action at $C_{\mathrm{SW}}=1.715$. These parameters correspond to the lattice spacing $a=0.0907(13) \mathrm{fm}$ and the spatial lattice volume $L^{3} \simeq(2.9 \mathrm{fm})^{3}$. The hopping parameters are taken to be $\kappa_{u d}=0.13700,0.13727$, 0.13754 for $u$ and $d$ quarks and $\kappa_{s}=0.13640$ for the $s$ quark. We employ the relativistic heavy quark action for the charm quark [28] to remove the leading order and next-to-leading order cutoff errors, $\mathcal{O}\left(\left(m_{c} a\right)^{n}\right)$ and $\mathcal{O}\left(\left(m_{c} a\right)^{n}\left(a \Lambda_{\mathrm{QCD}}\right)\right)$, respectively $[29,30]$. To improve the statistics, measurements are repeated twice for each configuration by shifting the source in time direction. The statistical errors are evaluated by the jackknife method. The calculated meson masses and the number of configurations $N_{\text {cfg }}$ used in our simulations are listed in Table I, together with the physical meson masses. The two-meson thresholds relevant to our analysis are shown in Fig. 1: Because of the heavy pion mass in our simulation, the $\pi \psi^{\prime}(3826)$ threshold is above the $\bar{D} D^{*}$ threshold. Also, $\rho \rightarrow \pi \pi$ decay is not allowed with $L \simeq 3 \mathrm{fm}$, so that $\rho \eta_{c}$ is a well-defined two-body channel. Pair annihilations of charm quarks are not considered in the present simulations.

In Fig. 2, we show the results of the $s$-wave $\pi J / \psi-\rho \eta_{c}-\bar{D} D^{*}$ coupled-channel potentials at time slice $t=13$, where the time-slice dependence in $t=11-15$ on the potentials $V^{\alpha \beta}$ is found to be weak: This implies that contributions from the inelastic $\bar{D}^{*} D^{*}$ scattering states to $V^{\alpha \beta}$ are negligible, and the convergence of the derivative expansion is reliable. We find that all diagonal potentials, (a) $V^{\bar{D} D^{*}, \bar{D} D^{*}}$, (c) $V^{\rho \eta_{c}, \rho \eta_{c}}$, and (f) $V^{\pi J / \psi, \pi J / \psi}$ are very weak. This observation indicates that the $Z_{c}(3900)$ is neither a simple $\pi J / \psi$ nor $\bar{D} D^{*}$ molecule. Among the off-diagonal 
TABLE I. Meson masses in MeV units and the number of configurations used in our simulations.

\begin{tabular}{lccccccr}
\hline \hline & $m_{\pi}$ & $m_{\rho}$ & $m_{\eta_{c}}$ & $m_{J / \psi}$ & $m_{\bar{D}}$ & $m_{D^{*}}$ & $N_{\mathrm{cfg}}$ \\
\hline Expt. & 140 & 775 & 2984 & 3097 & 1870 & 2007 & \\
Case I & $411(1)$ & $896(8)$ & $2988(1)$ & $3097(1)$ & $1903(1)$ & $2056(3)$ & 450 \\
Case II & $570(1)$ & $1000(5)$ & $3005(1)$ & $3118(1)$ & $1947(1)$ & $2101(2)$ & 400 \\
Case III & $701(1)$ & $1097(4)$ & $3024(1)$ & $3143(1)$ & $2000(1)$ & $2159(2)$ & 399 \\
\hline \hline
\end{tabular}

potentials, we find that the $\pi J / \psi-\rho \eta_{c}$ coupling in Fig. 2(e) is also weak: This is consistent with the heavy-quark spin symmetry, which tells us that the spin flip amplitudes of the charm quark are suppressed by $\mathcal{O}\left(1 / m_{c}\right)$. On the other hand, (b) the $\rho \eta_{c}-\bar{D} D^{*}$ coupling and (d) the $\pi J / \psi-\bar{D} D^{*}$ coupling are both strong: They correspond to the rearrangement of quarks between the hidden charm sector and the open charm sector.

As a first step to investigate the structure of the $Z_{c}(3900)$ on the basis of $V^{\alpha \beta}$ just obtained, let us consider the two-body $T$ matrix [see, e.g., Eq. (16.43) of Ref. [31]]:

$$
\begin{aligned}
& t^{\alpha \beta}\left(\vec{p}_{\alpha}, \vec{p}_{\beta} ; W_{\text {c.m. }}\right) \\
& =V^{\alpha \beta}\left(\vec{p}_{\alpha}, \vec{p}_{\beta}\right) \\
& \quad+\sum_{\gamma} \int d \vec{q}_{\gamma} \frac{V^{\alpha \gamma}\left(\vec{p}_{\alpha}, \vec{q}_{\gamma}\right) t^{\gamma \beta}\left(\vec{q}_{\gamma}, \vec{p}_{\beta} ; W_{\mathrm{c} . \mathrm{m} .}\right)}{W_{\mathrm{c} . \mathrm{m} .}-E_{\gamma}\left(\vec{q}_{\gamma}\right)+i \epsilon},
\end{aligned}
$$

where $\vec{p}_{\alpha}\left(\vec{q}_{\gamma}\right)$ indicates the on-shell (off-shell) momentum of the two-meson state in channel $\alpha(\gamma)$. $W_{\text {c.m. }}$, and $E_{\gamma}\left(\vec{q}_{\gamma}\right)$ represent the scattering energy in the center-of-mass (c.m.) frame and the energy of the intermediate states in channel $\gamma$, respectively.

Shown in Fig. 3(a) are the invariant mass spectra $\operatorname{Im} f^{\alpha \alpha}\left(W_{\text {c.m. }}\right)=-\pi \mu^{\alpha} \operatorname{Im} t^{\alpha \alpha}\left(W_{\text {c.m. }}\right)$ in the $\pi J / \psi$ (red circles), $\rho \eta_{c}$ (green triangles), and $\bar{D} D^{*}$ (blue squares) channels obtained from lattice QCD for case I in Table I. The amplitude $f^{\alpha \beta}\left(W_{\text {c.m. }}\right)$ is related to the differential cross section as $d \sigma^{\alpha \beta} / d \Omega=\left|f^{\alpha \beta}\left(W_{\text {c.m. }}\right)\right|^{2}$. In Fig. 3(a), the inner errors are statistical only, while the outer ones are statistical and systematic errors added in quadrature: The systematic errors from the truncation of the derivative expansion are evaluated by the difference between $\operatorname{Im} f^{\alpha \alpha}$ at $t=13$ and that at $t=15$. The peak structures in $\rho \eta_{c}$ and $\bar{D} D^{*}$ spectra are caused by the opening of the $s$-wave thresholds. The sudden enhancement in the $\pi J / \psi$ spectrum just above the $\bar{D} D^{*}$ threshold is induced by the $\pi J / \psi-\bar{D} D^{*}$ coupling. Indeed, if we switch off the off-diagonal components of $V^{\alpha \beta}$, the red circles turn into the black crosses without any peak structure. This implies that the peak structure in the $\pi J / \psi$ spectrum [called $Z_{c}(3900)$ ] is a typical "threshold cusp" [31,32] due to the opening of the $s$-wave $\bar{D} D^{*}$ threshold.
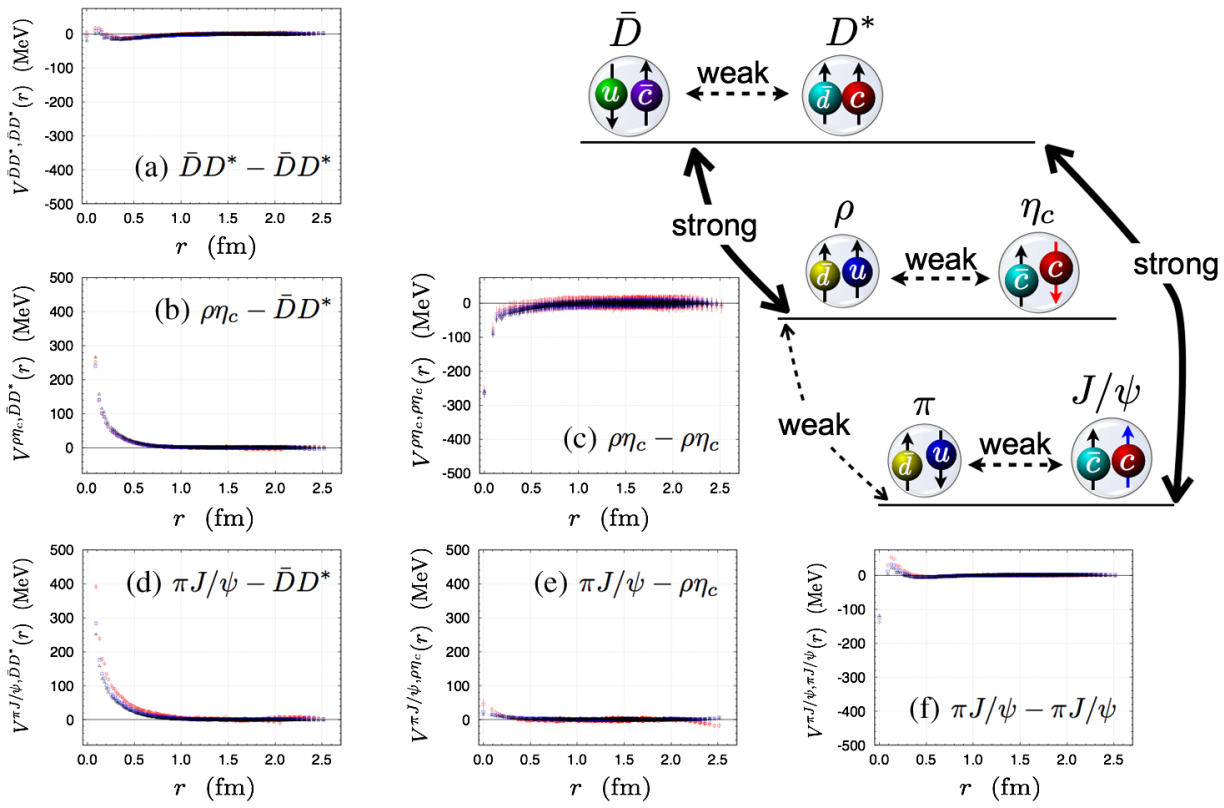

FIG. 2. The $s$-wave potentials for the (a) $\bar{D} D^{*}-\bar{D} D^{*}$, (b) $\rho \eta_{c}-\bar{D} D^{*}$, (c) $\rho \eta_{c}-\rho \eta_{c}$, (d) $\pi J / \psi-\bar{D} D^{*}$, (e) $\pi J / \psi-\rho \eta_{c}$, and (f) $\pi J / \psi-\pi J / \psi$ channels. The coupled-channel potentials are obtained at time slice $t=13$ for case I (red circles), case II (blue squares), and case III (black triangles). 

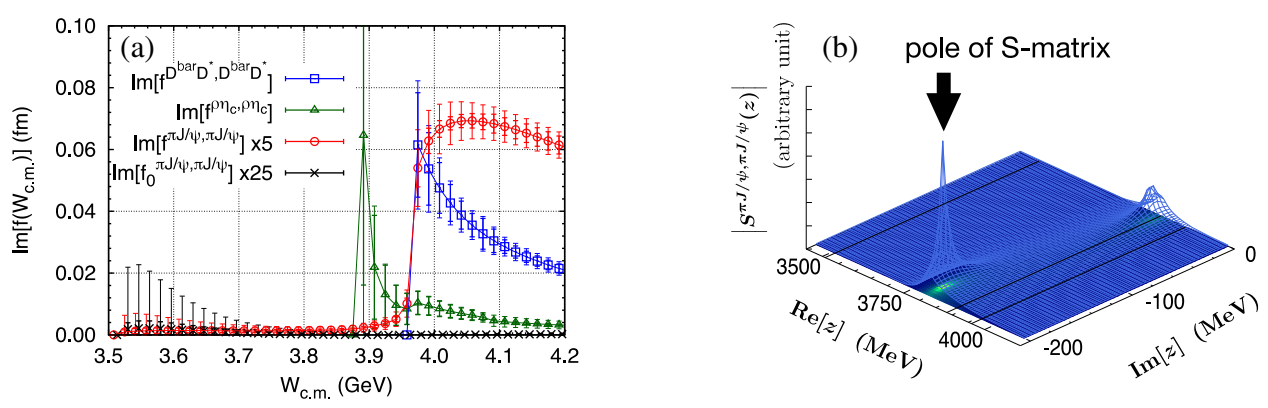

FIG. 3. (a) The two-body invariant mass spectra in the $\pi J / \psi$ (red circles, scaled by 5), $\rho \eta_{c}$ (green triangles), and $\bar{D} D^{*}$ (blue squares) channels. The two-body $\pi J / \psi$ spectrum without the off-diagonal component of $V^{\alpha \beta}$ is also shown by $\operatorname{Im} f_{0}^{\pi J / \psi, \pi J / \psi}$ (black crosses, scaled by 25). (b) The pole of the $S$ matrix on the [bbb] sheet in the notation of Ref. [33] for $\pi J / \psi, \rho \eta_{c}$, and $\bar{D} D^{*}$ channels $\left(z=m_{1}^{\alpha}+m_{2}^{\alpha}+p_{\alpha}^{2} / 2 \mu^{\alpha}\right)$. Both figures correspond to the case I in Table I. In Fig. 3(a), the inner error is statistical, while the outer one is statistical and systematic combined in quadrature.

To make sure that the $Z_{c}(3900)$ is not associated with the resonance structure, we examine the pole positions of the $S$ matrix on the complex energy plane according to the notation and procedure in Ref. [33]. The complex energy is defined as $z=m_{1}^{\alpha}+m_{2}^{\alpha}+p_{\alpha}^{2} / 2 \mu^{\alpha}$, and the "top $[t]$ " ("bottom $[b]$ ") sheet corresponds to $0 \leq \arg p_{\alpha}<\pi$ $\left(\pi \leq \arg p_{\alpha}<2 \pi\right)$ for the complex momentum in each channel $\left(\alpha=\pi J / \psi, \rho \eta_{c}, \bar{D} D^{*}\right)$. Among 8 Riemann sheets for the present three-channel scattering, the most relevant one is the $[b b b]$ sheet in the notation of Ref. [33]. We find a pole with a large imaginary part on this sheet (see Supplemental Material [34]): $z-\left(m_{\bar{D}}+\right.$ $\left.m_{D^{*}}\right)=-167(94)(27)-i 183(46)(19) \mathrm{MeV}$ for case $\mathrm{I}$, $-128(76)(33)-i 157(32)(19) \mathrm{MeV}$ for case II, and $-190(56)(42)-i 44(27)(27) \mathrm{MeV}$ for case III, with the first and second parentheses indicating the statistical and systematic errors, respectively. Shown in Fig. 3(b) is the complex pole on the [bbb] sheet for case I. It is located far from the $\bar{D} D^{*}$ threshold on the real axis, so that the amplitude is hardly affected by the pole.

To make further connection between the above result and the experimentally observed structure in $\pi J / \psi$ and $\bar{D} D^{*}$ invariant mass spectra [2-4], let us now consider semiphenomenological analysis of the three-body decays $Y(4260) \rightarrow \pi \pi J / \psi, \pi \bar{D} D^{*}$ by taking into account the final state rescattering due to $V^{\alpha \beta}$ extracted from lattice QCD simulations. We model the primary vertex by complex constants $C^{Y \rightarrow \pi+\alpha}\left[\alpha=\left(\pi J / \psi, \bar{D} D^{*}\right)\right]$. Then the threebody $T$ matrix $T^{Y \rightarrow \pi+\beta}\left[\beta=\left(\pi J / \psi, \bar{D} D^{*}\right)\right]$ is given by

$$
\begin{aligned}
& T^{Y \rightarrow \pi+\beta}\left(\vec{p}, \vec{q}_{\beta} ; W_{3}\right) \\
& =\sum_{\alpha=\pi J / \psi, \bar{D} D^{*}} C^{Y \rightarrow \pi+\alpha} \\
& \quad \times\left(\delta_{\alpha \beta}+\int d \vec{q}_{\alpha} \frac{t^{\alpha \beta}\left(\vec{q}_{\alpha}, \vec{q}_{\beta}, \vec{p} ; W_{3}\right)}{W_{3}-E_{\pi}(\vec{p})-E_{\alpha}\left(\vec{p}, \vec{q}_{\alpha}\right)+i \epsilon}\right),
\end{aligned}
$$

where $W_{3}, E_{\pi}(\vec{p})$, and $E_{\alpha}\left(\vec{p}, \vec{q}_{\alpha}\right)$ represent the energies of the $Y(4260)$, the spectator pion with the momentum $\vec{p}$ and the interacting pairs with the relative momentum $\vec{q}_{\alpha}$ in channel $\alpha$, respectively (see Supplemental Material [34]). The decay rate in the rest frame of $Y(4260)$ is obtained as $d \Gamma^{Y \rightarrow \pi+\beta}\left(W_{3}\right)=(2 \pi)^{4} \delta\left(W_{3}-E_{\pi}(\vec{p})-E_{\beta}\left(\vec{p}, \vec{q}_{\beta}\right)\right) d \vec{p} d \vec{q}_{\beta}$ $\left|T^{Y \rightarrow \pi+\beta}\left(\vec{p}, \vec{q}_{\beta} ; W_{3}\right)\right|^{2}$.

In order to have the same phase space as the experiments, we employ the physical hadron masses while $t^{\alpha \beta}$ is taken from the lattice data for case I. The complex couplings $C^{Y \rightarrow \pi+\alpha}$ are fitted to the BESIII data [2]. Since the experimental data are in the arbitrary scale, we focus only
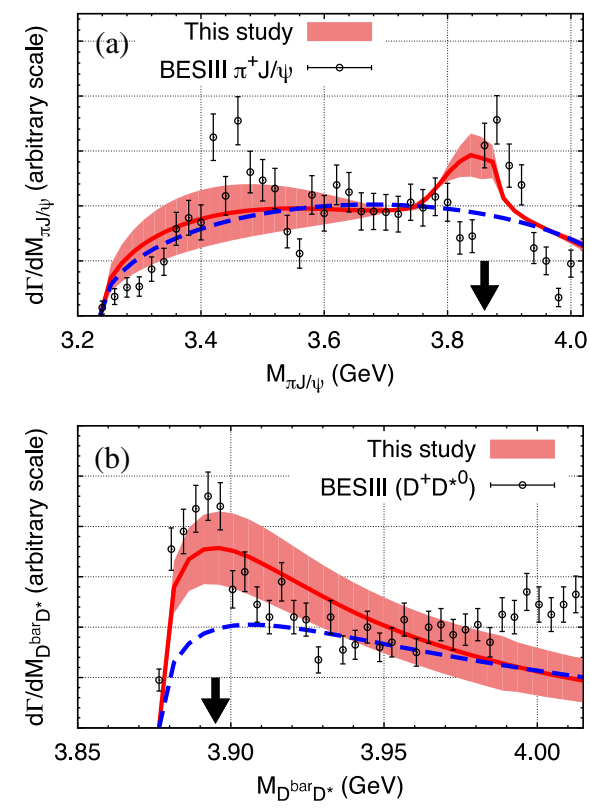

FIG. 4. The invariant mass spectra of (a) $Y(4260) \rightarrow \pi \pi J / \psi$ and (b) $Y(4260) \rightarrow \pi \bar{D} D^{*}$ below the $\bar{D}^{*} D^{*}$ threshold calculated with $V^{\alpha \beta}$ for case I in Table I. The shaded areas show the statistical errors. The vertical arrows show the predicted peak positions from the calculations. The blue dashed lines show the invariant mass spectra of the $Y(4260)$ decay without the offdiagonal components of $V^{\alpha \beta}$. The experimental data are taken from Ref. [2]. 
on the line shapes of the invariant mass spectra. In this case, we have two real parameters, $R \equiv\left|C^{Y \rightarrow \pi\left(\bar{D} D^{*}\right)} / C^{Y \rightarrow \pi(\pi J / \psi)}\right|$ and $\theta \equiv \arg \left(C^{Y \rightarrow \pi\left(\bar{D} D^{*}\right)} / C^{Y \rightarrow \pi(\pi J / \psi)}\right)$, and the best fit values are $R=0.95(18)$ and $\theta=-58(44)$ degree. To compare with the raw data of the experiment, we find $\mathcal{N}_{\pi J / \psi}\left|C^{Y \rightarrow \pi(\pi J / \psi)}\right|=0.40(22) \mathrm{GeV}^{-2}$ for the $\pi J / \psi$ and $\mathcal{N}_{\bar{D} D^{*}}\left|C^{Y \rightarrow \pi(\pi J / \psi)}\right|=0.76(42) \mathrm{GeV}^{-2}$ for the $\bar{D} D^{*}$ invariant mass distributions, where $\mathcal{N}_{\alpha}$ are the normalization factors to the raw data. Resulting decay spectra are shown in Figs. 4(a) and 4(b), where the shaded bands denote the statistical errors: We find that the coupled-channel potential $V^{\alpha \beta}$ well reproduces the peak structures just above the $\bar{D} D^{*}$ threshold at $3.9 \mathrm{GeV}$. The deviation from the experimental data around $4 \mathrm{GeV}$ may be attributed to the contributions from the higher partial waves between the spectator pion and interacting pairs or from the $\bar{D}^{*} D^{*}$ states, which are not considered in the present study. If we turn off the offdiagonal components of $V^{\alpha \beta}$ with the same constants $C^{Y \rightarrow \pi+\alpha}$, we obtain the results shown by the blue dashed lines, where the lines are normalized to the results obtained from the full calculations at $4 \mathrm{GeV}$. The peak structures at $3.9 \mathrm{GeV}$ disappear in this case.

In summary, we have studied the $\pi J / \psi-\rho \eta_{c}-\bar{D} D^{*}$ coupled-channel interactions using $(2+1)$-flavor full QCD gauge configurations in order to study the structure of the tetraquark candidate $Z_{c}(3900)$. Thanks to the HAL QCD method, we obtain the full coupled-channel potential $V^{\alpha \beta}$, whose diagonal components are all small, so that $Z_{c}(3900)$ cannot be a simple hadro-charmonium or $\bar{D} D^{*}$ molecular state.

Also, we found a strong off-diagonal transition between $\pi J / \psi$ and $\bar{D} D^{*}$, which indicates that the $Z_{c}(3900)$ can be explained as a threshold cusp. To confirm this, we calculated the invariant mass spectra and pole positions associated with the coupled-channel two-body $S$ matrix on the basis of $V^{\alpha \beta}$. The results indeed support that the peak in the $\pi J / \psi$ invariant mass spectrum is not associated with a conventional resonance state but is a threshold cusp induced by the strong $\pi J / \psi-\bar{D} D^{*}$ coupling. To further strengthen our conclusion, we made a semiphenomenological analysis of the three-body decay of the $Y(4260)$, and found that the experimentally observed peak structures just above the $\bar{D} D^{*}$ threshold are well reproduced in the $Y(4260) \rightarrow \pi \pi J / \psi$ and the $Y(4260) \rightarrow \pi \bar{D} D^{*}$ decays.

To make a definite conclusion on the structure of the $Z_{c}(3900)$ in the real world, we plan to carry out full QCD simulations near the physical point. It is also an interesting future problem to study the structure of pentaquark candidates $P_{c}^{+}(4380)$ and $P_{c}^{+}(4450)$ on the basis of the coupled-channel HAL QCD method.

The authors thank ILDG/JLDG [36] for providing us with full QCD gauge configurations used in this study. Y. I. is grateful to Doctor C.Z. Yuan for providing us with
BESIII experimental data. Numerical calculations were carried out on NEC-SX9 at Osaka University and SR 16000 at YITP in Kyoto University. This study is supported in part by JSPS KAKENHI Grants No. JP25800170, No. JP25287046, No. JP26400281, No. JP15K17667, and by MEXT as "Priority Issue on Post-K computer" (Elucidation of the Fundamental Laws and Evolution of the Universe) and SPIRE (Strategic Program for Innovative REsearch).

[1] R. Aaij et al. (LHCb Collaboration), Phys. Rev. Lett. 115, 072001 (2015).

[2] M. Ablikim et al. (BESIII Collaboration), Phys. Rev. Lett. 110, 252001 (2013); 112, 022001 (2014).

[3] Z. Q. Liu et al. (Belle Collaboration), Phys. Rev. Lett. 110, 252002 (2013).

[4] T. Xiao, S. Dobbs, A. Tomaradze, and K. K. Seth (CLEO-c Collaboration), Phys. Lett. B 727, 366 (2013).

[5] M. B. Voloshin, Phys. Rev. D 87, 091501 (2013).

[6] M. Cleven, F. K. Guo, C. Hanhart, Q. Wang, and Q. Zhao, Phys. Rev. D 92, 014005 (2015); M. Albaladejo, F.-K. Guo, C. Hidalgo-Duque, and J. Nieves, Phys. Lett. B 755, 337 (2016).

[7] D. Y. Chen, X. Liu, and T. Matsuki, Phys. Rev. D 88, 036008 (2013).

[8] E.S. Swanson, Phys. Rev. D 91, 034009 (2015); A. P. Szczepaniak, Phys. Lett. B 747, 410 (2015); E. S. Swanson, Int. J. Mod. Phys. E 25, 1642010 (2016).

[9] S. Prelovsek and L. Leskovec, Phys. Lett. B 727, 172 (2013); S. Prelovsek, C. B. Lang, L. Leskovec, and D. Mohler, Phys. Rev. D 91, 014504 (2015).

[10] S. H. Lee et al. (Fermilab Lattice and MILC Collaborations), Proc. Sci., LATTICE2014 (2014) 125.

[11] N. Ishii, S. Aoki, and T. Hatsuda, Phys. Rev. Lett. 99, 022001 (2007).

[12] S. Aoki, T. Hatsuda, and N. Ishii, Prog. Theor. Phys. 123, 89 (2010).

[13] N. Ishii, S. Aoki, T. Doi, T. Hatsuda, Y. Ikeda, T. Inoue, K. Murano, H. Nemura, and K. Sasaki (HAL QCD Collaboration), Phys. Lett. B 712, 437 (2012).

[14] S. Aoki et al. (HAL QCD Collaboration), Prog. Theor. Exp. Phys. 2012, 01A105 (2012).

[15] S. Aoki, N. Ishii, T. Doi, T. Hatsuda, Y. Ikeda, T. Inoue, K. Murano, H. Nemura, and K. Sasaki (HAL QCD Collaboration), Proc. Jpn. Acad. Ser. B 87, 509 (2011).

[16] S. Aoki, B. Charron, T. Doi, T. Hatsuda, T. Inoue, and N. Ishii, Phys. Rev. D 87, 034512 (2013).

[17] K. Sasaki, S. Aoki, T. Doi, T. Hatsuda, Y. Ikeda, T. Inoue, N. Ishii, and K. Murano (HAL QCD Collaboration), Prog. Theor. Exp. Phys. 2015, 113B01 (2015).

[18] M. Luscher, Nucl. Phys. B354, 531 (1991).

[19] T. Kurth, N. Ishii, T. Doi, S. Aoki, and T. Hatsuda, J. High Energy Phys. 12 (2013) 015.

[20] T. Iritani (HAL QCD Collaboration), Proc. Sci., LATTICE2015 (2016) 089.

[21] T. Iritani et al. (HAL QCD Collaboration), J. High Energy Phys. 10 (2016) 101. 
[22] K. Sasaki et al. (HAL QCD Collaboration), Proc. Sci., LATTICE2015, (2016) 088.

[23] M. Doring, U. G. Meissner, E. Oset, and A. Rusetsky, Eur. Phys. J. A 47, 139 (2011).

[24] J. J. Wu, T.-S. H. Lee, A. W. Thomas, and R. D. Young, Phys. Rev. C 90, 055206 (2014).

[25] J. J. Dudek, R. G. Edwards, C. E. Thomas, and D. J. Wilson (Hadron Spectrum Collaboration), Phys. Rev. Lett. 113, 182001 (2014).

[26] S. Aoki et al. (PACS-CS Collaboration), Phys. Rev. D 79, 034503 (2009).

[27] S. Aoki et al. (PACS-CS Collaboration), Phys. Rev. D 81, 074503 (2010).

[28] S. Aoki, Y. Kuramashi, and S.-i. Tominaga, Prog. Theor. Phys. 109, 383 (2003).

[29] Y. Namekawa et al. (PACS-CS Collaboration), Phys. Rev. D 84, 074505 (2011).
[30] Y. Ikeda, B. Charron, S. Aoki, T. Doi, T. Hatsuda, T. Inoue, N. Ishii, K. Murano, H. Nemura, and K. Sasaki (HAL QCD Collaboration), Phys. Lett. B 729, 85 (2014).

[31] R. G. Newton, Scattering Theory of Waves and Particles, 2nd ed. (Dover, New York, 2002).

[32] E. P. Wigner, Phys. Rev. 73, 1002 (1948).

[33] B. C. Pearce and B. F. Gibson, Phys. Rev. C 40, 902 (1989).

[34] See Supplemental Material at http://link.aps.org/ supplemental/10.1103/PhysRevLett.117.242001 for details about the pole positions and the derivation of the three-body amplitude for $Y(4260) \rightarrow \pi \pi J / \psi, \pi \bar{D} D^{*}$, which includes Refs. [33,35].

[35] K. A. Olive et al. (Particle Data Group), Chin. Phys. C 38, 090001 (2014), Sec. 46.4. (last reference in Supplemental Material [34], not already in Letter).

[36] International Lattice Data Grid, http://www.lqcd.org/ildg; Japan Lattice Data Grid, http://.jldg.org. 